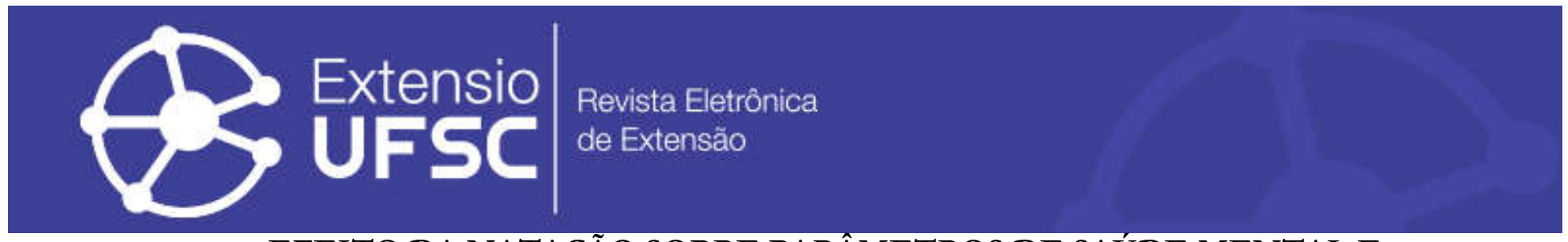

\title{
EFEITO DA NATAÇÃO SOBRE PARÂMETROS DE SAÚDE MENTAL E APTIDÃO FÍSICA FUNCIONAL EM ESCOLARES
}

\author{
Lorhan Menguer \\ Universidade do Extremo Sul Catarinense \\ lorhanmenguer@hotmail.com \\ Fernanda Sombrio \\ Universidade do Extremo Sul Catarinense \\ fe_sombrio@hotmail.com
}

\author{
Ramiro Doyenart Ferreira \\ Universidade do Extremo Sul Catarinense \\ ramadoyenart@gmail.com \\ Karin Martins Gomes \\ Universidade do Extremo Sul Catarinense \\ karin@unesc.net \\ Luciano Acordi da Silva \\ Universidade do Extremo Sul Catarinense \\ luciano_acordi@unesc.net
}

Resumo

Objetivo: Investigar o efeito de um programa de extensão universitária que oferece a modalidade de natação para crianças sobre escores de saúde mental e aptidão física funcional. Método: Estudo transversal, quantitativo com 33 crianças, de idades entre 10 a 12 anos, participantes do projeto de Extensão Golfinhos entre março e junho de 2017, sendo randomizadas em grupo praticantes de natação $(n=18)$ e grupo não praticante $(n=15)$. Resultados: Nossos achados demonstram reduções significativas $(\mathrm{p}<0.005)$ no grupo praticante de natação nos escores de hiperatividade, depressão, ansiedade e aumentos na agilidade, quando comparado ao grupo não praticante. Conclusão: Apontamos que crianças praticantes de natação apresentam melhor saúde mental e aptidão física funcional, quando comparadas a crianças não praticantes.

Palavras-chave: Crianças. Hiperatividade. Ansiedade. Natação.

\section{EFFECT OF SWIMMING ON MENTAL HEALTH PARAMETERS AND FUNCTIONAL PHYSICAL FITNESS IN CHILD STUDENTS}

\begin{abstract}
Objective: To investigate the effect of a swimming program on mental health and functional physical fitness scores in schoolchildren. Method: Cross-sectional, quantitative study with 33 children, aged 10 to 12 years, participating in the Dolphin Extension project between March and June 2017, being randomized into a group of swimmers $(\mathrm{n}=18)$ and a non-swimmer group $(\mathrm{n}=15)$. Results: Our findings demonstrate significant reductions $(\mathrm{p}<0.005)$ in the swimming group in the scores of hyperactivity, depression, anxiety and increases in agility, when compared to the non-practicing group. Conclusion: We point out that children who practice swimming have better mental health and functional physical fitness when compared to non-practicing children.
\end{abstract}

Keywords: Children. Hyperactivity. Anxiety. Swimming.

\section{EFECTO DE LA NATACIÓN EN LOS PARÁMETROS DE SALUD MENTAL Y EN LA APTITUD FÍSICA FUNCIONAL EN ESTUDIANTES INFANTILES}

\section{Resumen}

Objetivo: Investigar el efecto de un programa de natación en la salud mental y en las puntuaciones de aptitud física funcional en escolares. Método: estudio cuantitativo transversal con 33 niños, de entre 10 y 12 años, que participaron en el proyecto Dolphins Extension entre marzo y junio de 2017, y se asignaron al azar a un grupo de practicantes de natación (n $=18)$ y a un grupo no practicante $(n=15)$ Resultados: Nuestros hallazgos demuestran reducciones significativas ( $p$ $<0.005$ ) en el grupo de natación en los puntajes de hiperactividad, depresión, ansiedad y aumentos en la agilidad, en comparación con el grupo no practicante. Conclusión: Señalamos que los niños que practican la natación tienen mejor salud mental y aptitud física funcional en comparación con los niños que no practican.

Palavras clave: Niños. Hiperactividad. Ansiedad. Natación. 
Efeito da natação sobre parâmetros de saúde mental e aptidão física funcional em escolares

\section{INTRODUÇÃO}

Atualmente, as universidades têm assumido um papel, de aproximar as comunidades com suas atividades de extensão, que articulam ensino e pesquisa, em prol de uma sociedade mais humana e saudável. Neste sentido, oferecer projetos gratuitos que ajudem a melhorar a saúde mental de crianças e adolescentes em situações vulneráveis, através da prática de exercício físico, é importante.

Estima-se que de 10 a $20 \%$ da população infantil mundial é afetada por problemas na saúde, incluindo transtorno de hiperatividade, ansiedade e depressão (SCHULTE-KÖRNE, 2016). Crianças com esses transtornos, apresentam sintomas de desatenção, irritabilidade, dificuldade de aprendizado, isolamento social, baixo rendimento escolar e muitas vezes abandono (SCHULTE-KÖRNE, 2016; KOVACS et al., 1989).

Neste sentido, os níveis inapropriados de condicionamento físico em crianças podem interferir negativamente, aumentando os transtornos supracitados (WU et al., 2017; MINISTÉRIO DA SAÚDE, 2005). Alterações afetando a capacidade intelectual, a inserção social e o condicionamento físico importantes para promoção da saúde são cada vez mais comuns em crianças e adolescentes sedentários (GUERRA, JÚNIOR e FLORINDO, 2006).

Segundo pesquisa nacional de saúde, feita pelo Instituto Brasileiro de Geografia e Estatística (IBGE, 2010), a prevalência de adolescentes escolares sedentários é alta no Brasil (78\% no total, sendo $79,2 \%$ para o sexo feminino e 76,7\% para o sexo masculino). Kovacs et al. (1989) e Wu et al. (2017) afirmaram, que o sedentarismo infantil pode prejudicar a saúde mental das crianças e, consequentemente, aumentar os índices de reprovação escolar, desistência e abandono. Contudo, no Brasil, ainda são poucos os programas de exercícios físicos direcionados para o melhoramento da saúde coletiva, relacionadas à saúde mental em escolares sedentários (MINISTÉRIO DA SAÚDE, 2005).

Todavia, diferentes estudos reportam que pessoas acometidas de disfunções mentais beneficiam-se na promoção da saúde, quando utilizam o exercício físico como ferramenta coadjuvante em seus tratamentos (FARIOLI VECCHIOLI et al., 2018; BIDDLE, 2016). É fato que a prática de exercício físico regular, com destaque para exercícios aeróbios, traz inúmeros benefícios para a promoção da saúde (LUDYGA et al., 2017; DELEVATTI et al., 2018). Sabe-se que a prática de natação, caracterizado como exercício aeróbio, é fonte de inúmeros benefícios, tais como a melhora na aptidão aeróbia, muscular e motora (RODRIGUES e LIMA, 2014; CONTI, 2015; DORMEHL et al., 2017). Contudo, a literatura ainda é escassa sobre o efeito da natação sobre parâmetros de saúde mental em crianças. 
Efeito da natação sobre parâmetros de saúde mental e aptidão física funcional em escolares

Partindo desses pressupostos, o objetivo do presente estudo foi investigar o efeito de um programa de Extensão Universitária que oferece a modalidade de natação para crianças e adolescentes, sobre os parâmetros de saúde mental e aptidão física funcional em escolares.

\section{MATERIAIS E MÉTODOS}

\section{Considerações éticas}

Este estudo foi aprovado pelo comitê de ética local (parecer 1.305.625; CAE 50245515.0.0000.0119), sendo realizado de acordo com as diretrizes e normas regulamentadoras de pesquisa envolvendo seres humanos (466/12). Todos os pais e/ou responsáveis assinaram o termo de consentimento esclarecido autorizando a participação das crianças como voluntárias à pesquisa.

\section{Projeto de Extensão Golfinhos}

Fundado em vinte e um de abril de 2016, o projeto de extensão Golfinhos tem o objetivo de ensinar crianças do Bairro da Juventude de Criciúma a nadar os quatro estilos olímpicos de nado e praticar os esportes coletivos aquáticos, biribol e pólo aquático. O Golfinhos é realizado na piscina da Universidade do Extremo Sul Catarinense (UNESC), no período da tarde, duas vezes por semana. Atende em média 50 crianças por semestre. Alunos da graduação, com orientação do docente responsável pelo projeto, ministram as aulas para as crianças. Paralelamente, o projeto alimenta os cursos de educação física e psicologia, amparando estágios, desenvolvendo pesquisas de conclusão de curso e projetos de iniciação científica da Universidade.

\section{Desenho}

Trata-se de um estudo transversal, de caso controle quantitativo (MEDRONHO, 2009), com crianças praticantes do projeto de Golfinhos, envolvendo a modalidade de natação versus crianças não praticantes. Todas frequentantes da mesma escola pública da cidade, que tem parceria com nossa Universidade. Quarenta e oito horas após a última sessão de natação, as crianças foram submetidas a testes de saúde mental e aptidão física e avaliadas posteriormente.

\section{Participantes}

A população do estudo foi composta por 225 crianças que frequentavam a mesma escola. A amostral foi selecionada por critério de conveniência não probabilística, sendo composto por trinta e três crianças de ambos os sexos, com idade entre 10 e 12 anos, alocadas em grupo não 
Efeito da natação sobre parâmetros de saúde mental e aptidão física funcional em escolares

praticante $(n=15)$ e grupo praticante de natação $(n=18)$, através de sorteio (tabela 1). Para obtenção do número amostral significativo, foi utilizado como base um estudo similar envolvendo adolescentes nadadores (DA SILVA; et al., 2019).

Tabela 1. Caraterização da amostra

$\begin{array}{ccc}\begin{array}{c}\text { Não Praticantes } \\ (\mathbf{n}=\mathbf{1 5})\end{array} & \text { Praticantes Natação } & \text { valor } \mathbf{p} \\ 7 \text { meninas } & 9 \text { meninas } & \\ 8 \text { meninos } & 9 \text { meninos } & 0.991 \\ 11 \pm 2 \text { anos } & 12 \pm 2 \text { anos } & 0.892 \\ \end{array}$

Tempo de prática natação não se aplica $\quad 4 \pm 1$ meses

Frequência semanal não se aplica 1 vezes

Duração da aula não se aplica $\quad 45 \mathrm{mim}$

Nível de aprendizagem não se aplica Iniciantes

Nota: Não houve diferença significativa entre os grupos ( $p>0.05)$. Fonte: Os autores.

Sobre os critérios de inclusão, foram adotados: a) apresentar indicação médica para a prática de natação; b) estar frequentando regularmente a escola; c) não apresentar nenhuma limitação física ou psicológica que pudesse comprometer os resultados. Em relação aos critérios de exclusão, foram adotados: a) crianças que praticavam outro esporte ou atividade física paralela; b) que não trouxeram o termo de consentimento assinado pelos pais; c) que durante o programa faltaram mais do que $30 \%$ as sessões de natação.

\section{Procedimentos}

O grupo praticante de natação realizou o programa que consistiu de 12 semanas com frequência semanal de uma vez, e tempo de aula de 45 minutos. As aulas eram divididas em: 5 min de alongamento, 10 min de atividade recreativa de adaptação ao meio liquido, 25 min como parte principal, com quatro a cinco exercícios de aprendizagem de natação, e, na parte final, que consistia de $5 \mathrm{mim}$, as crianças nadavam livre sem imposição de velocidade e finalizavam com alongamento. Em média, as crianças nadavam 500 metros por aula. A intensidade da aula foi monitorada através de percepção subjetiva de esforço (BORG, 2000), em que as crianças eram aconselhadas a nadar na intensidade de 11 a 13 pontos da escala. As aulas de natação ocorreram na piscina térmica semiolímpica da Universidade do Extremo Sul Catarinense (UNESC). 
Efeito da natação sobre parâmetros de saúde mental e aptidão física funcional em escolares

O grupo não praticante não realizou nenhum esporte ou atividade física regular durante o estudo, apresentando as mesmas características do grupo natação. Ambos os grupos foram avaliados 48 horas após a última sessão de aula do programa de natação. Todos os testes foram aplicados em nas dependências da UNESC no mesmo horário e dia.

$\mathrm{Na}$ aplicação dos inventários foi dado um tempo de 15 minutos para cada inventário. Durante a aplicação, as crianças foram acompanhadas pelos pesquisadores, que permaneceram na sala para sanar todas as dúvidas e garantir a individualidade nas respostas.

Para análise da hiperatividade foi aplicado o inventário Swanson, Nolan and Pelham Teacher and Parent Rating Scale (SNAP-IV), construído a partir dos sintomas do manual de diagnóstico e estatística Diagnostic and Statistical Manual of Mental Disorders (DSM-IV), da Associação Americana de Psiquiátrica (DSM-IV, 1995). De acordo com suas diretrizes, para responder o questionário, foi solicitado a dois professores da escola que conviviam com as crianças que respondessem o mesmo. Sobre a avaliação dos sintomas de ansiedade e depressão, foi utilizado o Inventário Beck de ansiedade e depressão, traduzidos para língua portuguesa e validado para população brasileira de acordo com Gorenstein (GORENSTEIN e ANDRADE, 1996)

Após esses procedimentos, as crianças foram direcionadas para a quadra da instituição para realização dos testes físicos. Em relação aos parâmetros de aptidão física funcional, aplicaram-se os testes de agilidade, flexibilidade e resistência abdominal proposto por Gaya (2015). Especificamente, para quantificação dos escores de agilidade foi aplicado o teste do quadrado; para flexibilidade, o teste de sentar e alcançar e, para resistência muscular do tronco, o teste de resistência abdominal.

\section{Análise estatística}

Posteriormente os dados foram analisados utilizando o software SPSS 20.0 para Windows. Foi utilizado o teste $T$ de Student, não pareado para analisar as diferenças significativas entre as médias do grupo praticante versus não praticantes. O nível de significância adotado foi de $\mathrm{p}<$ 0,05 e os dados foram expressos em média e \pm desvio padrão (DP).

\section{RESULTADOS DISCUSSÃO}

É fato que os exercícios predominantemente aeróbios, como a natação, são recomendados para a promoção e manutenção da saúde (CONTI, 2015; DORMEHL, 2017; POWERS e HOWLEY, 2014). O presente estudo aponta que o programa de extensão universitária Golfinhos melhorou cientificamente os escores de saúde mental em escolares, 
Efeito da natação sobre parâmetros de saúde mental e aptidão física funcional em escolares

concretizando uma articulação importante entre a universidade e a sociedade. Destacamos, felizmente, que esta atividade extesionista (projeto Golfinhos), é um exemplo impulsionador indispensável, para consolidação do tripé atual universitário (ensino/pesquisa/extensão).

Sendo assim, em relação aos escores de hiperatividade em crianças em idade escolar (Figura 1), os resultados do presente projeto de extensão demostram que através da prática de natação é possível reduzir este escore. O estudo de Ludyga et al. (2017) apontaram que uma única sessão de 20 minutos de exercício aeróbio de moderada intensidade pode ter implicações positivas sobre aspectos cognitivos e controle inibitório em crianças hiperativas. Bois et al. (2005) reportam que a prática de atividade física pode aumentar a autoestima, a aceitação social e a sensação de bem-estar entre as crianças.

Figura 1: Escores de Hiperatividade em crianças de Criciúma, Santa Catarina, Brasil, 2017.

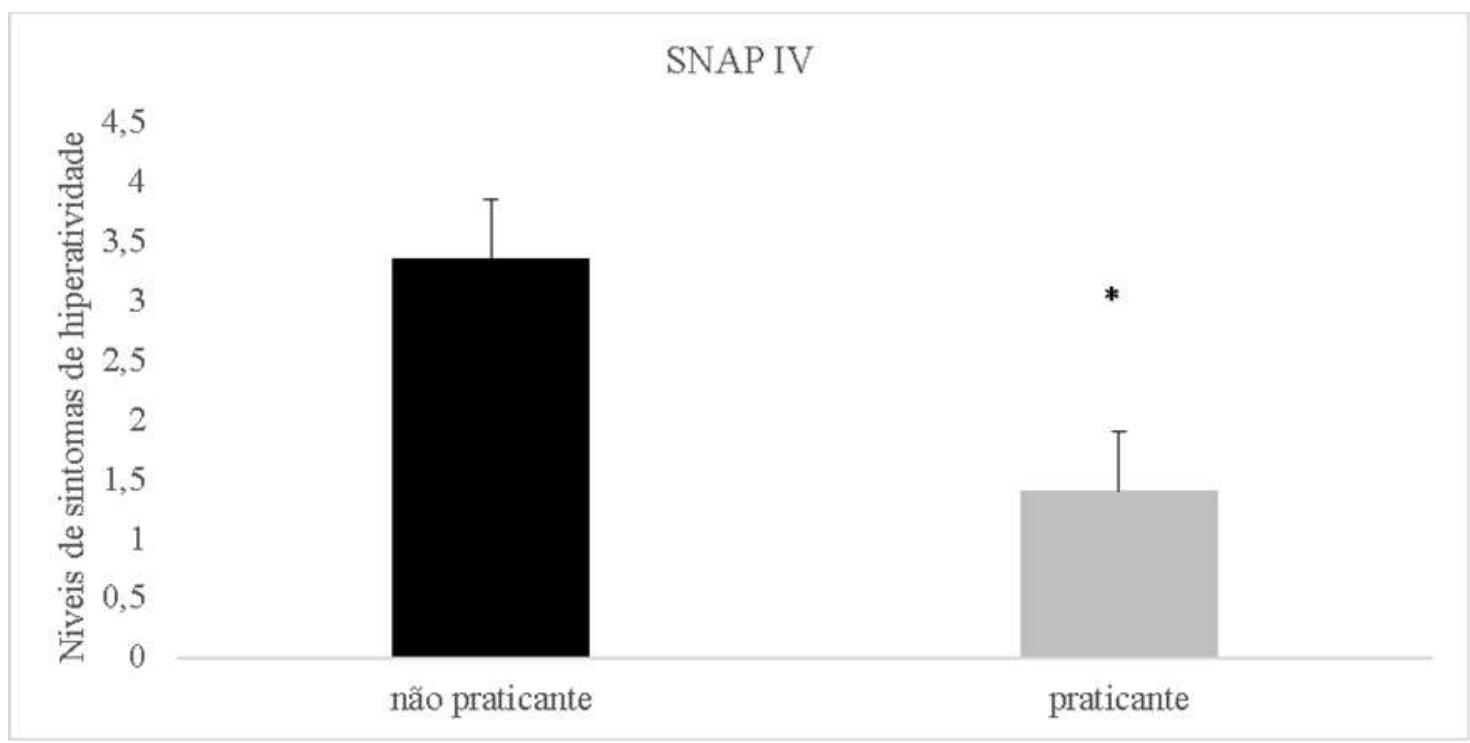

Nota: Parâmetro de Hiperatividade em crianças não praticantes e praticantes de natação com idade entre 10 e 12 anos. Diferença significativa $\mathrm{p}=0.047$. Fonte: Os autores.

Seguindo este raciocínio, estudos vêm demostrando que a realização de exercícios físicos de forma isolada ou combinada com outras intervenções (fármacos e terapias) vem ajudando a modular positivamente parâmetros bioquímicos e clínicos em diversas situações patológicas de doenças crônicas do sistema nervoso central (DELEVATTI, 2018). Especificamente, o exercício físico aeróbio pode modular funções cognitivas importantes. Em outro estudo, realizado no mesmo laboratório, apontou-se que crianças com TDAH praticantes de natação de um programa de oito semanas melhoraram significativamente a plasticidade cognitiva, assim como a atenção seletiva (DA SILVA, 2019). 
Efeito da natação sobre parâmetros de saúde mental e aptidão física funcional em escolares

Como segundo parâmetro, nós avaliamos os escores de depressão em crianças. O presente estudo demonstra que o grupo praticante de natação apresenta menores escores de depressão, quando comparado ao não praticante (Figura 2).

Figura 2. Níveis de Depressão em crianças de Criciúma, Santa Catarina, Brasil, 2017.

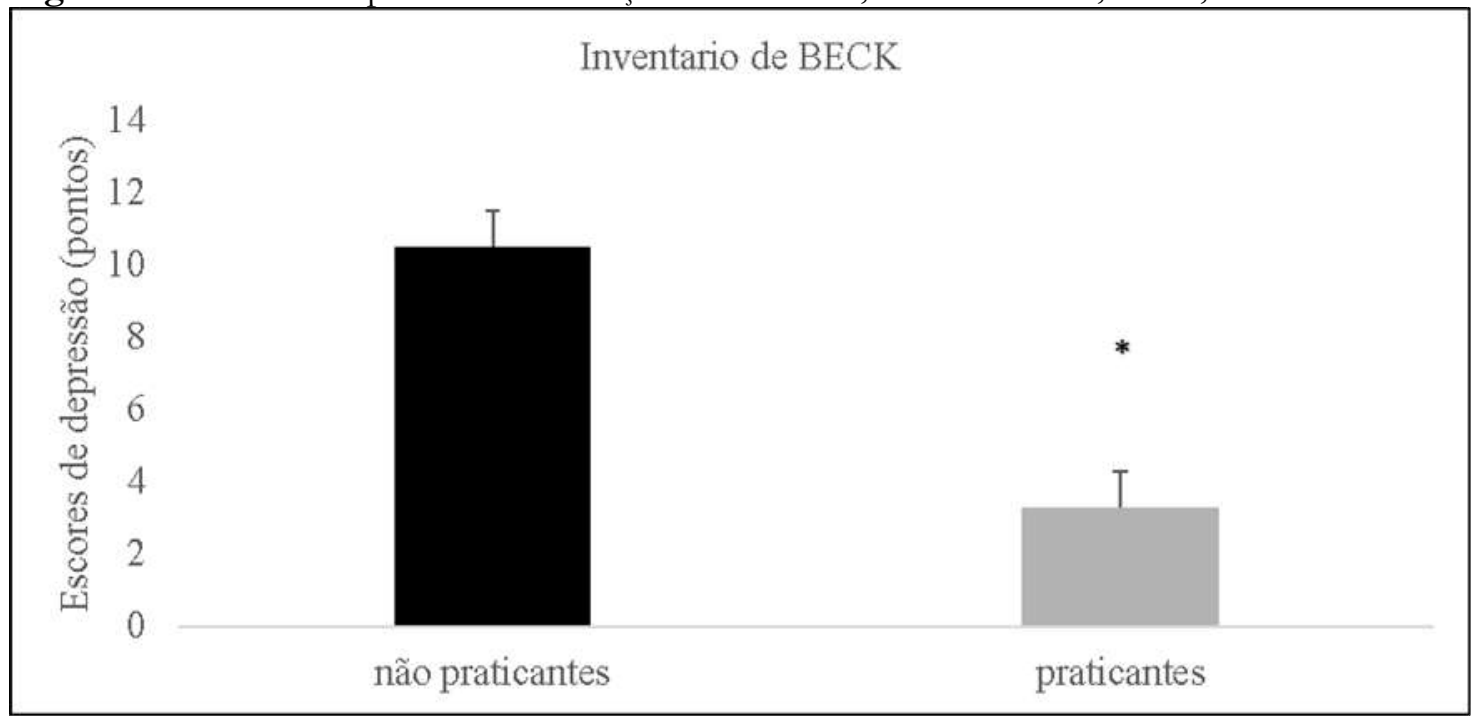

Nota: Parâmetro de Depressão em crianças não praticantes e praticantes de natação com idade entre 10 e 12 anos Diferença significativa $\mathrm{p}<0.049$. Fonte: Os autores.

Estudos envolvendo exercícios físicos regulares têm apontado efeitos antidepressivos via rotas psicofisiológicas (REBAR et al., 2015; MIKKELSEN et al., 2017). Algumas destas rotas têm sido ativadas por aumento do fluxo sanguíneo cerebral, da atividade do eixo hipotálamopituitária-adrenal em diferentes regiões do cérebro, incluindo o sistema límbico, a amigdala e o hipocampo, assim como o aumento da autoeficácia e dos pensamentos positivos (BIDDLE, 2016). Yu et al. (2013) reportam que o exercício aeróbio de moderada intensidade (três vezes por semana, durante um período de seis meses) reduziu os níveis de depressão em idosos, devido ao aumento do nível de endorfinas, que agem sobre o sistema nervoso (MIKKELSEN et al., 2017; ROELANDS, DE PAUW e MEEUSEN, 2015). No estudo realizado por Silva et al. (2019), crianças com TDAH reduziram significativamente os níveis de depressão após um programa de oito semanas de natação. Segundo os autores, o exercício físico modula o sistema dopaminérgico, que está associado às regulações de humor e sintomas depressivos.

Como terceiro parâmetro de saúde mental, nós avaliamos a ansiedade. A ansiedade é caracterizada por tensão ou desconforto derivado de antecipação de perigo, algo desconhecido ou estranho (ZARRINDAST e KHAKPAI, 2015). Em escolares, quando não tratada, pode deixar cicatrizes profundas na vida das crianças, permanecendo até a vida adulta (CASTILLO et 
Efeito da natação sobre parâmetros de saúde mental e aptidão física funcional em escolares

al., 2000). O presente estudo demostrou que crianças praticantes de natação apresentam menores sintomas de ansiedade quando comparadas ao grupo não praticante (Figura 3).

Figura 3: Níveis de Ansiedade em crianças de Criciúma, Santa Catarina, Brasil, 2017.

\section{Inventario de BECK}

\section{2}

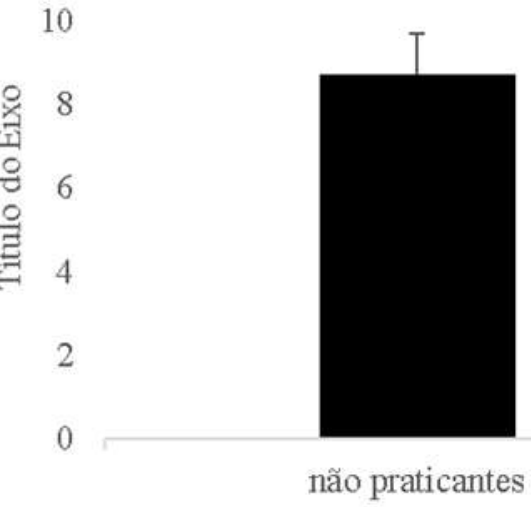

Nota: Parâmetro de Ansiedade em crianças não praticantes e praticantes de natação com idade entre 10 e 14 anos. Diferença significativa $\mathrm{p}<0.01$. Fonte: Os autores.

Diversos estudos demostram que os exercícios alteram sintomas de ansiedade (MCMAHON et al., 2017; WEGNER et al., 2014). Da Silva et al. (2018) apontam que o exercício aquático de baixa intensidade reduz sintomas de ansiedade. Broocks et al. (1998) revisaram a literatura sobre os efeitos neurobiológicos do exercício físico, sugerindo que existem evidências experimentais suficientes para apontar que o exercício regular induz a uma regulação de certos receptores serotoninérgicos centrais, que desempenham um papel importante na patogênese da ansiedade. A realização de exercícios físicos resulta em uma série de alterações fisiológicas e bioquímicas envolvidas, com a liberação de neurotransmissores e ativação de receptores específicos (MIKKELSEN et al., 2017; ROELANDS, DE PAUW e MEEUSEN, 2015; ZARRINDAST e KHAKPAI, 2015), que auxiliaram na diminuição dos escores indicativos de ansiedade. Estas alterações podem ajudar a explicar como o exercício físico utilizado neste estudo reduziu os sintomas de ansiedade.

Em relação à aptidão física funcional, avaliou-se os níveis de agilidade, sendo este definido por Powers e Howley (2014) como a valência física que possibilita mudar a posição do corpo ou a direção do movimento, no menor tempo possível. Os resultados demostraram que a agilidade dos praticantes de natação é maior do que os não praticantes (Figura 4). 
Efeito da natação sobre parâmetros de saúde mental e aptidão física funcional em escolares

Figura 4: Capacidade funcional em crianças de Criciúma, Santa Catarina, Brasil, 2017.

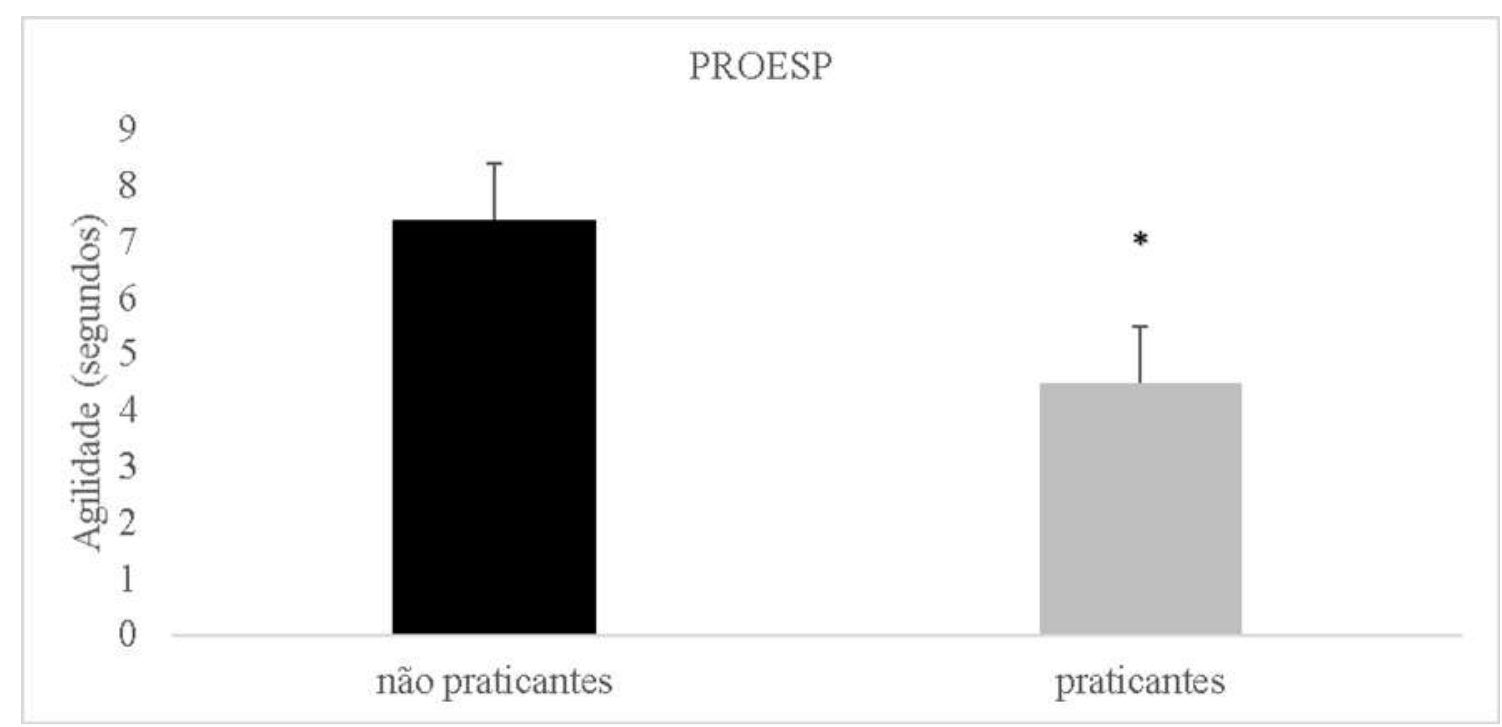

Nota: Parâmetro de capacidade funcional, mensurado através do teste de agilidade em crianças não praticantes e praticantes de natação com idade entre 10 e 12 anos. Diferença significativa p=0.039.

Fonte: Os autores.

O estudo de Khodaei, Mohammadi e Badri (2017) aponta para aumento significativo na agilidade após a prática de exercícios pliométricos. Antunes et al. (2001) demostraram que indivíduos submetidos a exercícios físicos, três vezes por semana, durante seis meses, obtiveram melhoras na agilidade. De acordo com os resultados do presente estudo, observou-se que o programa de natação melhorou a agilidade. Logo, acredita-se que isso ocorreu devido às características dos movimentos coordenativos motores inerentes à natação, que são: coordenação de braços (braçada), pernas (pernada) e respiração em outro plano (transversal). A execução do movimento de braçada, pernada e respiração são consideradas complexas, já que acontecem de maneira simultânea. Isso acaba exigindo que a criança amplie o seu repertório motor, executando os movimentos com uma carga de complexidade psicofisiológica maior, o que, por sua vez, contribui para a melhoria da agilidade.

Outros dois parâmetros de aptidão física avaliados, foram a resistência abdominal e a flexibilidade. A resistência abdominal e a flexibilidade são funcionalmente importantes para saúde, pois os componentes motores envolvidos nessas habilidades modulam o sistema musculoesquelético, que tem um papel indispensável na realização das atividades diárias (GUEDES e GUEDES, 1997; GLANER, 2003). Os resultados apontam que não houve diferença significativa em ambos os parâmetros. Por outro lado, da Silva et al. (2019) reportaram melhoras na resistência abdominal e flexibilidade de crianças após uma programada de natação com frequência semanal de duas vezes, durante oito semanas. Acredita-se que a ausência de 
Efeito da natação sobre parâmetros de saúde mental e aptidão física funcional em escolares

melhoria sobre estes dois parâmetros no grupo praticante de natação, neste estudo, pode ser explicada em decorrência da baixa frequência semanal (uma vez) de aula.

Tabela 2. Resistência abdominal e flexibilidade em crianças de Criciúma, Santa Catarina, Brasil, 2017.

\section{Não praticante $\quad$ Praticante $\quad$ Valor $p$}

\begin{tabular}{llll}
\hline Resistência abdominal (repetições) & $28.7 \pm 6.8$ & $33.4 \pm 9.5$ & 0.078
\end{tabular}

$\begin{array}{llll}\text { Flexibilidade }(\mathrm{cm}) & 24.5 \pm 6.2 & 27 \pm 6.8 & 0.088\end{array}$

Nota: Valores médios resistência abdominal e flexibilidade em crianças não praticantes e praticantes de natação com idade entre 10 e 12 anos. Não houve diferença significativa em relação ao controle respectivamente. p>0,05.

Fonte: Os autores.

Por fim, salientamos a importância dessa ação extensionista (projeto Golfinhos) como forma de acesso para a população, oferecendo gratuitamente a prática orientada de atividade física. Também é admirável elucidar o projeto, como campo de estágio no curso de educação física e psicologia, articulando a promoção do esporte, associado à saúde mental, impulsionado pelos acadêmicos. Sugerimos que este tipo de ação se emancipe, tonando-se permanente em nossa universidade, entendendo que o esporte é mais uma mola propulsora. Todavia é necessário um olhar multiprofissional envolvendo psicólogos, sociólogos, médicos, enfermeiros, nutricionistas, assistentes sociais, entre outras áreas, a fim de combater deterioração da saúde mental, em função dos novos tempos (tecnológicos, pandêmicos, hipocinéticos), que estamos passando.

\section{CONSIDERAÇÕES FINAIS}

Em suma, aponta-se que crianças praticantes de natação do projeto de extensão universitária Golfinhos apresentaram melhores escores de saúde mental e aptidão física funcional, quando comparadas aos não praticantes. Acredita-se que crianças com transtornos de ansiedade e hiperatividade podem ser beneficiadas, melhorando a saúde mental através da prática da natação e, consequentemente, também o aprendizado escolar. Contudo, apontamos como limitação a ausência das avaliações de base, sendo necessários estudos longitudinais, com maior rigor científico e profundidade, sobre essa temática. Todavia, destacamos que o projeto de Extensão Golfinhos é mais uma ferramenta pedagógica interessante que vem sendo desenvolvida na Universidade local, ancorado no tripé atual, que é fazer ensino, pesquisa e extensão de forma articulada. 
Efeito da natação sobre parâmetros de saúde mental e aptidão física funcional em escolares

\section{REFERÊNCIAS}

ANTUNES, Hanna K. M.; et al. Alterações cognitivas decorrentes do exercício físico sistematizado. Revista da Sobama, 6, 27-33, 2001.

BIDDLE, Stuart. Physical activity and mental health: evidence is growing. World Psychiatry, 15(2), 176-177, 2016.

BOIS, Julien; et al. Elementary schoolchildren's perceived competence and physical activity involvement: The influence of parents' role modeling behaviors and perceptions of their child's competence. Psychology of Sport and Exercise, 6(4), 381-397, 2005.

BORG, Gunnar. Escalas de Borg para a dor e o esforço percebido. São Paulo: Manole, 2000. BRASIL. MINISTÉRIO DA SAUUDE; SECRETARIA DE ATENÇÃO À SAÚDE. Reforma psiquiátrica e política de saúde mental no brasil. Documento apresentado à Conferência Regional de Reforma dos Serviços de Saúde Mental: 15 anos depois de Caracas. Brasília: Ministério da Saúde, 2005.

BRASIL. MINISTÉRIO DO PLANEJAMENTO, ORÇAMENTO E GESTÃO. Instituto Brasileiro de Geografia e Estatística. Pesquisa de orçamentos familiares 2008-2009: antropometria e estado nutricional de crianças, adolescentes e adultos no Brasil. Rio de Janeiro: Instituto Brasileiro de Geografia e Estatística (IBGE), 2010.

BROOCKS, Andreas; et al. Comparison of aerobic exercise, clomipramine, and placebo in the treatment of panic disorder. The American Journal of Psychiatry, 155(5), 603-609, 1998.

Castillo, Ana Regina; et al. Transtornos de ansiedade. Revista Brasileira de Psiquiatria, 22(2), 20-23, 2000.

CONTI, A. A. Nuoto, attività fisica e salute: una prospettiva storica. Clinical Therapeutics; 166(4), 179-182, 2015.

DA SILVA, Luciano Acordi; et al. Swimming training improves mental health parametres, cognition and motorcoor dination in children with Attention Deficit Hyperactivity Disorder. International Journal of Environmental Health Research, 13(1), 1-9, 2019.

DA SILVA, Luciano Acordi; et al. Effect of aquatic exercise on mental health, functional autonomy, and oxidative dysfunction in hypertensive adults. Clinical and Experimental Hypertension, 40(6), 547-553, 2018.

DELEVATTI, Rodrigo S.; et al. Quality of life and sleep quality are similarly improved after aquatic or dry-land aerobic training in patients with type 2 diabetes: A randomized clinical trial. Journal of Science and Medicine in Sport, 21(5), 483-488, 2018.

DORMEHL, Shilo J.; et al. Confirming the Value of Swimming-Performance Models for Adolescents. International Journal of Sports Physiology and Performance,12(9), 1177-1185, 2017.

DSM-IV. Manual diagnóstico e estatístico de transtornos mentais. 4. ed. Porto Alegre: Artes Médicas, 1995. 
Efeito da natação sobre parâmetros de saúde mental e aptidão física funcional em escolares

FARIOLI-VECCHIOLI, Stefano; et al. The Role of Physical Exercise and Omega-3 Fatty Acids in Depressive Illness in the Elderly. Current Neuropharmacology, 16(3), 308-326, 2018.

GAYA, A. C. A. Projeto esporte Brasil: manual de testes e avaliação. Porto Alegre: UFRGS, 2015.

GLANER, M. F. Importância da aptidão física relacionada a saúde. Revista Brasileira de Cineantropometria \& Desempenho Humano, 5(2), 75-85, 2003.

GORENSTEIN, C.; ANDRADE, L. H. Validation of a Portuguese version of Beck Depression Inventory and the State-Trait Anxiety Inventory in Brazilian subjects. Brazilian Journal of Medical and Biological Research, 29(4), 453-457, 1996.

GUEDES, D.P.; GUEDES, J.E.R.P. Crescimento, composição corporal e desempenho motor. São Paulo: CLR Balieiro, 1997.

GUERRA, Paulo Henrique; JÚNIOR, José Cazuza de Farias; FLORINDO, Alex Antônio. Comportamento sedentário em crianças e adolescentes brasileiros: revisão sistemática. Revista de Saúde Pública, 1(1), 50-59, 2016.

KHODAEI, Kazem; MOHAMMADI, Abbas; BADRI, Neda. A comparison of assisted, resisted, and common plyometric training modes to enhance sprint and agility performance. The Journal of Sports Medicine and Physical Fitness, 57(10), 1237-1244, 2017.

KOVACS, Maria; et al. Depressive disorders in childhood: IV. A longitudinal study of comorbidity with and risk for anxiety disorders. Archives of General Psychiatry, 46(9), 776782, 1989.

LUDYGA, Sebastian; et al. An event-related potential investigation of the acute effects of aerobic and coordinative exercise on inhibitory control in children with ADHD._Developmental Cognitive Neuroscience, 28(17), 21-28, 2017.

MCMAHON, Elaine M.; et al. Physical activity in European adolescents and associations with anxiety, depression and well-being. European Child \& Adolescent Psychiatry, 26(1), 111122, 2017.

MEDRONHO, Roberto A. Epidemiologia. 2. ed. São Paulo: Atheneu, 2009.

MIKKELSEN, Kathleen; et al. Exercise and mental health. Maturitas, 106(1), 48-56, 2017.

POWERS, Scott K.; HOWLEY, Edward T. Fisiologia Do Exercício: teoria e aplicação ao condicionamento a ao desempenho. Barueri: Manole, 2014.

REBAR, Amanda L.; et al. A meta-meta-analysis of the effect of physical activity on depression and anxiety in non-clinical adult populations. Health Psychology Review, 9(3), 366-378, 2015.

RODRIGUES, M. N.; LIMA, S. R. Atividades motoras aquáticas na coordenação corporal de adolescentes com deficiência intelectual. Revista Brasileira de Ciências do Esporte, 36(2), 369-381, 2014. 
Efeito da natação sobre parâmetros de saúde mental e aptidão física funcional em escolares

ROELANDS, B.; DE PAUW, K., MEEUSEN, R. Neurophysiological effects of exercise in the heat. Scandinavian Journal of Medicine \& Science in Sports, 25(1), 65-78, 2015.

SCHULTE-KÖRNE, Gerd. Mental Health Problems in a School Setting in Children and Adolescents. Deutsches Ärzteblatt International, 113(11), 183-190, 2016.

WEGNER, Mirko; et al. Effects of exercise on anxiety and depression disorders: review of metaanalyses and neurobiological mechanisms. CNS \& Neurological Disorders Drug Targets, 13(6), 1002-1014, 2014.

WU, Xiu Yun; et al. The influence of physical activity, sedentary behavior on health-related quality of life among the general population of children and adolescents: A systematic review. Plos On,12(11), 1-29, 2017.

YU, Fang; et al. Affecting cognition and quality of life via aerobic exercise in Alzheimer's disease. Western Journal of Nursing Research, 35(1), 24-38, 2013.

ZARRINDAST, Mohammad-Reza; KHAKPAI, Fatemeh. The Modulatory Role of Dopamine in Anxiety-like Behavior. Archives of Iranian Medicine,18(9), 591-603, 2015.

Recebido em: 06/04/2020

Aceito em: 08/03/2021 